\title{
The magnetoelectric domains and cross-field switching in multiferroic $\mathrm{BiFeO}_{3}$
}

\author{
L. J. Li, ${ }^{1}$ J. Y. Li, ${ }^{1, a)}$ Y. C. Shu, ${ }^{2}$ and J. H. Yen ${ }^{2}$ \\ ${ }^{1}$ Department of Mechanical Engineering, University of Washington, Seattle, Washington 98195-2600, USA \\ ${ }^{2}$ Institute of Applied Mechanics, National Taiwan University, Taipei 106, Taiwan, Republic of China
}

(Received 26 September 2008; accepted 23 October 2008; published online 11 November 2008)

$\mathrm{BiFeO}_{3}$ is an exciting multiferroic material because of its room temperature multiferrocity, excellent ferroelectric properties, and recently demonstrated electric control of antiferromagnetic domains. In this letter we report a theoretical study on the structure and evolution of magnetoelectric domains in $\mathrm{BiFeO}_{3}$. We not only observed the coupled ferroelectric and antiferromagnetic domains and demonstrated the electric control of antiferromagnetic ordering, both in consistency with experiments, but also revealed the switching of antiferromagnetic domains by mechanical stress that is yet to be explored in experiments. (C) 2008 American Institute of Physics.

[DOI: $10.1063 / 1.3025843$ ]

Magnetoelectric coupling in multiferroic materials is of renewed interest not only for its fundamental scientific significance, ${ }^{1-7}$ but also for its potential applications in data storage and spintronics. ${ }^{3-7}$ Among all multiferroic materials, $\mathrm{BiFeO}_{3}$ is particularly exciting for its rare room temperature multiferrocity. ${ }^{8,9}$ It has a rhombohedral crystalline structure, with spontaneous polarization along one of the eight pseudocubic [111] axes. $^{10,11}$ In addition, it has G-type antiferromagnetic ordering, with magnetic spins lying in the (111) plane, being ferromagnetically coupled within the (111) plane while antiferromagnetically coupled between adjacent (111) planes. ${ }^{12,13}$ The correlation between the directions of polarizations and spins makes it possible to control the magnetic spins of $\mathrm{BiFeO}_{3}$ by electric fields, which has been demonstrated in experiments recently. ${ }^{4,6,14,15}$

The rapid experimental advances in $\mathrm{BiFeO}_{3}$ are exciting, but many questions remain to be explored. For example, what is the structure of magnetoelectric domains in $\mathrm{BiFeO}_{3}$, and how does its domain structure evolve under an external stimulus? Answers to these questions are essential to the understanding of the microstructure and macroscopic properties of $\mathrm{BiFeO}_{3}$, yet no theoretical framework has been developed to study the structure and evolution of magnetoelectric domains in $\mathrm{BiFeO}_{3}$ or any other multiferroics, and only the ferroelectric domain structure of $\mathrm{BiFeO}_{3}$ has been simulated so far. $^{16,17}$

In this letter, we apply an unconventional phase field approach recently developed ${ }^{18-21}$ to study the coupled magnetoelectric domains and cross-field switching in $\mathrm{BiFeO}_{3}$. Four order parameters are required to describe the multiferroic state of $\mathrm{BiFeO}_{3}$, including transformation strain $\mathbf{e}$, spontaneous polarization $\mathbf{p}$, and spins $\mathbf{m}_{a}$ and $\mathbf{m}_{b}$ of two sublattices that are antiferromagnetically coupled. The rhombohedral crystalline structure of $\mathrm{BiFeO}_{3}$ results in eight ferroelectric variants, with the transformation strain and spontaneous polarization given by

\footnotetext{
a) Author to whom correspondence should be addressed. Electronic mail: jjli@u.washington.edu.
}

$$
\mathbf{e}^{(1,2)}=\left[\begin{array}{lll}
\alpha & \delta & \delta \\
\delta & \alpha & \delta \\
\delta & \delta & \alpha
\end{array}\right], \quad \mathbf{p}^{(1,2)}= \pm \frac{p_{s}}{\sqrt{3}}\left[\begin{array}{l}
1 \\
1 \\
1
\end{array}\right]
$$

and their symmetry permutations, where $\mathbf{p}^{(3,4)} \| \pm[\overline{1} 11]$, $\mathbf{p}^{(5,6)} \| \pm[1 \overline{1} 1]$, and $\mathbf{p}^{(7,8)} \| \pm[\overline{1} \overline{1} 1]$. Notice that the strain and polarization of the ferroelectric can be determined from the volume averaging of its eight variants, $\mathbf{e}=\sum_{i=1}^{8} \lambda_{i} \mathbf{e}^{(i)}, \mathbf{p}$ $=\sum_{i=1}^{8} \lambda_{i} \mathbf{p}^{(i)}$, where $\lambda_{i}$ is the global volume fraction of variant $i$ satisfying $\sum_{i=1}^{8} \lambda_{i}=1$. To incorporate this constraint, we introduce seven $\mu_{i}$ that are independent of each other, with

$$
\lambda_{r}= \begin{cases}\mu_{r} \prod_{i=1}^{r-1}\left(1-\mu_{i}\right), & r=1, \ldots, 7, \\ r-1 & \\ \prod_{i=1}^{r}\left(1-\mu_{i}\right), & r=8,\end{cases}
$$

whose summation equals 1 , so that the constraint on $\lambda_{i}$ is automatically satisfied. Physically, $\mu_{i}$ can be interpreted as the characteristic functions of a multirank laminated microstructure, ${ }^{2-24}$ being either 0 or 1 as enforced by the first term in Eq. (3). This establishes the equivalence of characteristic functions $\boldsymbol{\mu}=\left\{\mu_{i}\right\}_{i=1}^{7}$ with the strain e and polarization $\mathbf{p}$, allowing us to use them as the internal variables. Indeed, the energetic contribution from the ferroelectric ordering is postulated as

$$
W_{e}^{\mathrm{ani}}(\boldsymbol{\mu})+W_{e}^{\mathrm{ex}}(\boldsymbol{\mu})=K_{e} \sum_{i=1}^{7} \mu_{i}^{2}\left(1-\mu_{i}\right)^{2}+A_{e}|\nabla \boldsymbol{\mu}|^{2},
$$

where the first term ensures the multiwell energetic structure minimized by $\mu_{i}$ at either 0 or 1 , and the second term accounts for the ferroelectric domain wall energy. Furthermore, there are elastic and electrostatic energies arising from the interactions among ferroelectric variants, which can be determined by solving the mechanical equilibrium equation ${ }^{18,19}$ and Maxwell's equation. ${ }^{25}$

The internal energy of $\mathrm{BiFeO}_{3}$ is also dependent on spins $\mathbf{m}_{a}$ and $\mathbf{m}_{b}$ of two sublattices, leading to additional energetic contribution from antiferromagnetic ordering, 


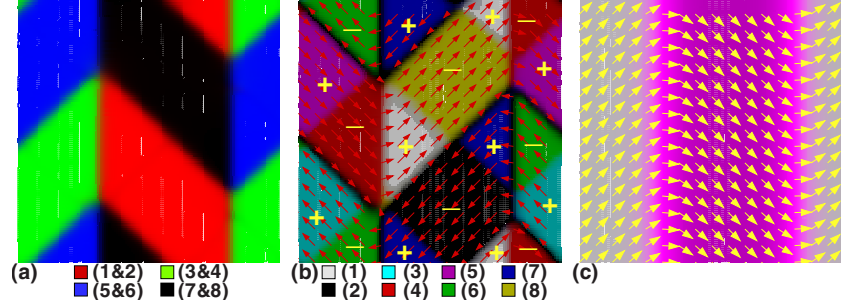

FIG. 1. (Color online) The configuration of (a) ferroelastic, (b) ferroelectric, and (c) antiferromagnetic domains of a bulk $\mathrm{BiFeO}_{3}$ in the absence of external fields projected on the (001) plane.

$$
W_{m}^{\mathrm{af}}\left(\mathbf{m}_{a}, \mathbf{m}_{b}\right)=A_{m}\left(\left|\nabla \mathbf{m}_{a}\right|-\left|\nabla \mathbf{m}_{b}\right|\right)^{2}-C \mathbf{m}_{a} \cdot \mathbf{m}_{b},
$$

where the first term accounts for the domain wall energy of spins, and the second term ensures that $\mathbf{m}_{a}$ and $\mathbf{m}_{b}$ are antiferromagnetically coupled. Magnetic spins lie in the (111) plane that is perpendicular to the [111] axis, the direction of polarization, and a magnetic anisotropy energy is introduced to reflect this coupling,

$$
W_{m}^{\text {ani }}\left(\mathbf{m}_{a}, \mathbf{m}_{b}, \boldsymbol{\mu}\right)=\frac{K_{m}}{2}\left\{2-\left[\mathbf{m}_{a} \cdot \mathbf{l}(\boldsymbol{\mu})\right]^{2}-\left[\mathbf{m}_{b} \cdot \mathbf{l}(\boldsymbol{\mu})\right]^{2}\right\},
$$

where $\mathbf{l}=\sum_{i=1}^{8} \lambda_{i^{(}}{ }^{(i)}$ gives the easy axis of spins, with $\mathbf{I}^{(1,2,7,8)}$ $=[1 \overline{1} 0]$ and $\mathbf{l}^{(3,4,5,6)}=[110]$, consistent with experimental observations and first principles computations on $\mathrm{BiFeO}_{3}$ films. ${ }^{4,13}$ The magnetization at an arbitrary material point thus is given by $\mathbf{m}=\sum_{i=1}^{8} \lambda_{i}\left(\mathbf{m}_{a}^{(i)}+\mathbf{m}_{b}^{(i)}\right)$, leading to additional magnetostatic energy that can be determined by solving Maxwell's equation. ${ }^{26}$ Notice that we couple the spins with the ferroelectric ordering through the anisotropy energy in Eq. (5) instead of rigidly as we do for the strain and polarization in Eq. (1) since it allows the rotation of spins that is often observed in magnetic materials. ${ }^{21}$

Through the variation in the potential energy, the evolution equations for $\boldsymbol{\mu}, \mathbf{m}_{a}$, and $\mathbf{m}_{b}$ are established as

$$
\begin{aligned}
& \frac{\partial \boldsymbol{\mu}}{\partial t}=L\left[\mathbf{F}^{\mathrm{ex}}+\mathbf{F}_{e}^{\mathrm{ani}}+\mathbf{F}_{m}^{\mathrm{ani}}+\mathbf{F}^{\mathrm{elas}}+\mathbf{F}^{\mathrm{ele}}\right], \\
& \frac{\partial \mathbf{m}_{a, b}}{\partial t}=-\gamma_{g} \mathbf{m}_{a, b} \times \mathbf{H}_{a, b}^{\mathrm{eff}}-\delta \gamma_{g} \mathbf{m}_{a, b} \times\left(\mathbf{m}_{a, b} \times \mathbf{H}_{a, b}^{\mathrm{eff}}\right),
\end{aligned}
$$

where the second equation describes the evolution of $\mathbf{m}_{a}$ or $\mathbf{m}_{b}$ in the form of the classical Landau-Lifshitz-Gilbert equation. $^{26} \quad$ In these equations, $\quad \mathbf{F}^{\mathrm{ex}}=2 A_{e} \nabla^{2} \boldsymbol{\mu}, \quad \mathbf{F}_{e}^{\text {ani }}$ $=(-\partial / \partial \boldsymbol{\mu}) W_{e}^{\mathrm{ani}}(\boldsymbol{\mu}), \quad \mathbf{F}_{m}^{\mathrm{ani}}=-(\partial / \partial \boldsymbol{\mu}) W_{m}^{\mathrm{ani}}\left(\mathbf{m}_{a}, \mathbf{m}_{b}, \boldsymbol{\mu}\right) \quad$ that couples the transformation strain and the magnetization, $\mathbf{F}^{\text {elas }}=\mathbf{C}[\boldsymbol{\varepsilon}-\mathbf{e}(\boldsymbol{\mu})] \cdot(\partial \mathbf{e}(\boldsymbol{\mu}) / \partial \boldsymbol{\mu})$, with $\mathbf{C}$ being the elastic stiffness tensor and $\boldsymbol{\varepsilon}$ the total strain, and $\mathbf{F}^{\text {ele }}=\left(\mathbf{E}^{0}\right.$ $-\nabla \phi) \cdot(\partial \mathbf{p} / \partial \boldsymbol{\mu})$, with $\mathbf{E}^{0}$ being the applied electric field and $-\nabla \phi$ the electrostatic field. ${ }^{25}$ The effective magnetic field is given by

$$
\mathbf{H}_{a, b}^{\mathrm{eff}}=\mathbf{H}_{a, b}^{e}+\mathbf{H}_{a, b}^{a}+\mathbf{H}^{0}-\nabla \psi,
$$

where $\mathbf{H}_{a, b}^{e}=\left(2 A_{m} / M_{s}\right)\left(\nabla^{2} \mathbf{m}_{a, b}-\nabla^{2} \mathbf{m}_{b, a}\right)+\left(C / M_{s}\right) \mathbf{m}_{b, a}, \mathbf{H}_{a, b}^{a}$ $=-\left(1 / M_{s}\right)\left(\partial / \partial \mathbf{m}_{a, b}\right) W_{m}^{\text {ani }}\left(\mathbf{m}_{a}, \mathbf{m}_{b}, \boldsymbol{\mu}\right), \mathbf{H}^{0}$ is the applied magnetic field, and $-\nabla \psi$ is the magnetostatic field. ${ }^{27}$ These equations are then solved by semi-implicit finite difference method and fast Fourier transform. ${ }^{21,28,29}$ The material pa-
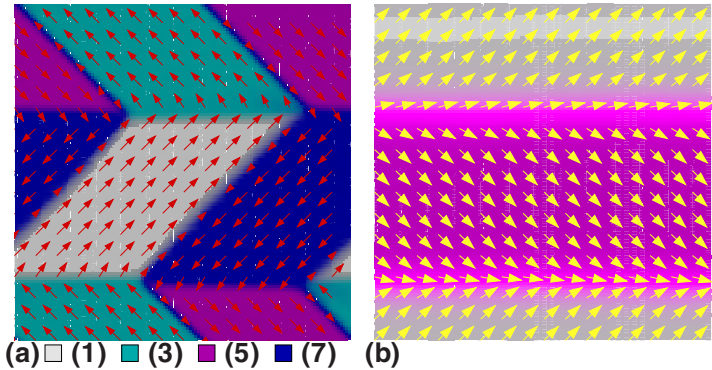

(b)
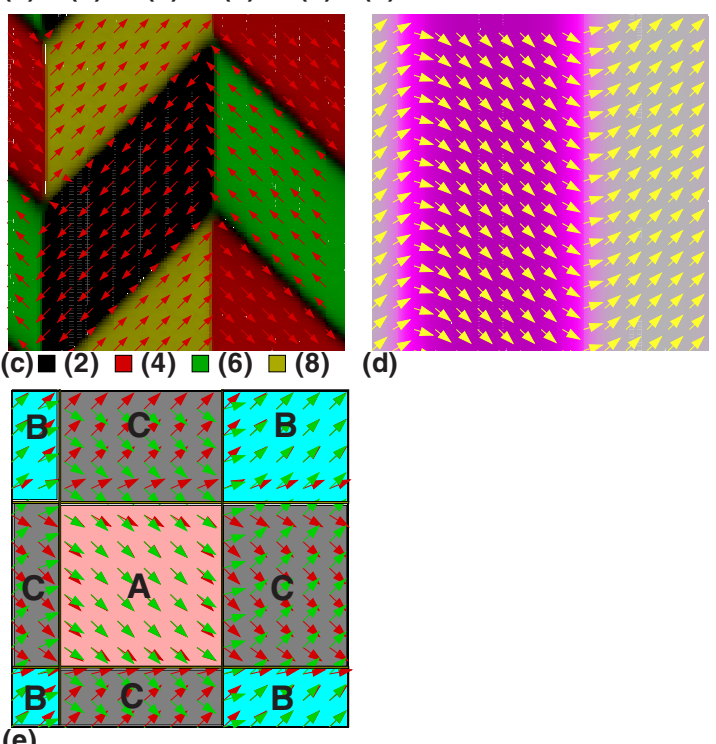

(e)

FIG. 2. (Color online) The configuration of ferroelectric and antiferromagnetic domains of $\mathrm{BiFeO}_{3}$ thin film oriented along the [001] axis; (a) ferroelectric and (b) antiferromagnetic domains under an external electric field along the [001] axis; (c) ferroelectric and (d) antiferromagnetic domains under an external electric field along the [001] axis; and (e) schematics showing switching of antiferromagnetic domains resulting from ferroelectric switching, with red arrows indicating spin directions before switching and green arrows indicating spin directions after switching.

rameters used in simulation include the following: elastic constants $C_{11}=302 \mathrm{GPa}, C_{12}=162 \mathrm{GPa}$, and $C_{44}=68 \mathrm{GPa}$ and magnetic anisotropic constant $K_{m}=6.6 \times 10^{4} \mathrm{~J} / \mathrm{m}^{3}$.

We first examine the ferroelastic, ferroelectric, and antiferromagnetic domain structures of $\mathrm{BiFeO}_{3}$ in the absence of external fields, as shown in Fig. 1. It is observed that four ferroelastic variants are arranged in a characteristic rank-2 laminate structure, which is further divided into eight ferroelectric domains in a self-accommodating and compatible matter. The arrows in Fig. 1(b) indicate the directions of in-plane polarization components, while the directions of out-of-plane components are indicated by + and - signs. Only two antiferromagnetic variants are observed, with their spins oriented along [110] and [1 $\overline{1} 0]$, as indicated by the yellow arrow in Fig. 1(c). The direction of sublattice spin $\mathbf{m}_{a}$ is shown, while the sublattice spin $\mathbf{m}_{b}$ is antiparallel to $\mathbf{m}_{a}$. It is noted that ferroelectric variants separated by $180^{\circ}$ [variants $(1) /(2),(3) /(4),(5) /(6),(7) /(8)]$ or $109^{\circ}$ [variants $(1) /(8),(2) /(7),(3) /(6)$, and $(4) /(5)]$ domain walls share the same antiferromagnetic ordering, while variants $(1) /(4)$ and (6)/(7) are separated by $71^{\circ}$ domains walls that change the antiferromagentic ordering, suggesting the possibility of electric control of antiferromagnetic ordering through the corresponding $109^{\circ}$ ferroelectric domain switching. 


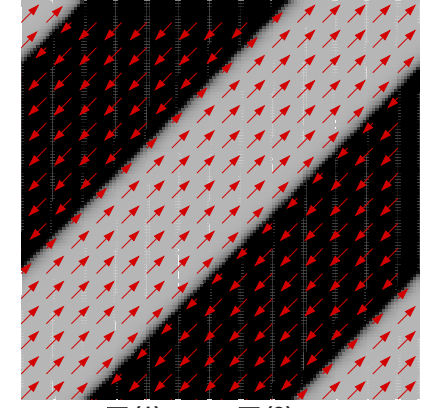

(a)

$\square(1)$

(2)

(b)

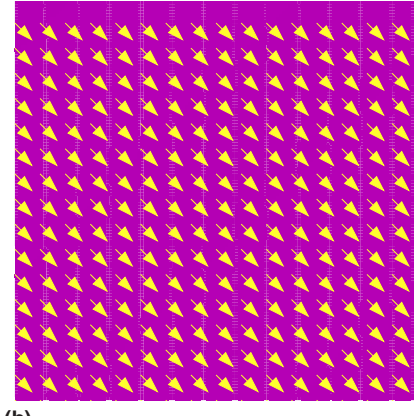

(b)

FIG. 3. (Color online) The configuration of ferroelectric and antiferromagnetic domains of $\mathrm{a} \mathrm{BiFeO}_{3}$ thin film oriented along the [001] axis; (a) ferroelectric domains and (b) antiferromagnetic domain under a mechanical shear stress $\sigma_{12}$; the directions of polarizations and spins projected onto the (001) plane are indicated by arrows in the respective figures.

To verify the electric control of antiferromagnetic domains, we apply an electric field along the [001] axis to a $\mathrm{BiFeO}_{3}$ film, and a new set of ferroelectric and antiferromagnetic domains emerges, as shown in Figs. 2(a) and 2(b). We then apply an opposite electric field along the [001] axis, which switches both ferroelectric and antiferromagnetic domains, as shown in Figs. 2(c) and 2(d). Interestingly, such $180^{\circ}$ polarization reversal is not achieved through $180^{\circ}$ domain switching, as one might expect, since it may lead to incompatibility among variants and thus is energetically disfavored. Instead, both $109^{\circ}$ and $71^{\circ}$ domain switchings are observed, leading to a new rank-2 laminated structure for ferroelectric domains that appear to result from a $90^{\circ}$ rotation of an original ferroelectric domain structure, consistent with lateral piezoresponse force microscopy (PFM) observation as reported in Figs. 5(c) and 5(d) of Zhao et al. ${ }^{4}$ The apparent $90^{\circ}$ rotation in ferroelectric domains also leads to $90^{\circ}$ rotation in antiferromagnetic domains, as shown in Figs. 2(b) and 2(d), and three regimes in ferroelectric switching can be identified, as shown in Fig. 2(e), where A and B denote regions of $180^{\circ}$ and $71^{\circ}$ ferroelectric switchings that do not change the antiferromagnetic ordering, while $\mathrm{C}$ denotes region of $109^{\circ}$ ferroelectric switching that switches the antiferromagnetic ordering.

The correlation of magnetic spins and crystalline structure also raises an interesting question if it is possible to switch the antiferromagnetic domains by mechanical stress. By applying a shear stress $\sigma_{12}$ to a domain structure similar to Figs. 2(a) and 2(b), one of the ferroelastic variants disappears, leaving behind only one remaining ferroelastic variant that has a uniform distribution of antiferromagnetic ordering, yet is divided into two ferroelectric domains, as shown in Fig. 3 This confirms that the antiferromagnetic domain can indeed be switched by a mechanical stress, offering additional control over the multiferroic ordering.
In summary, we developed a theoretical framework to study the coupled magnetoelectric domains and cross-field switching in multiferroic $\mathrm{BiFeO}_{3}$. Our study provides insight into the microstructure evolution and macroscopic properties of $\mathrm{BiFeO}_{3}$ and offers a powerful tool to analyze a wide range of multiferroic materials with magnetoelectric coupling.

We acknowledge support from US NSF (DMR 0706100), ARO (W911NF-07-1-0410), and TW NSC (972221-E-002-125-MY3).

${ }^{1}$ M. Fiebig, T. Lottermoser, D. Frohlich, A. V. Goltsev, and R. V. Pisarev, Nature (London) 419, 818 (2002).

${ }^{2}$ J. Wang, J. B. Neaton, H. Zheng, V. Nagarajan, S. B. Ogale, B. Liu, D. Viehland, V. Vaithyanathan, D. G. Schlom, U. V. Waghmare, N. A. Spaldin, K. M. Rabe, M. Wuttig, and R. Ramesh, Science 299, 1719 (2003).

${ }^{3}$ W. Eerenstein, N. D. Mathur, and J. F. Scott, Nature (London) 442, 759 (2006)

${ }^{4}$ T. Zhao, A. Scholl, F. Zavaliche, K. Lee, M. Barry, A. Doran, M. P. Cruz, Y. H. Chu, C. Ederer, N. A. Spaldin, R. R. Das, D. M. Kim, S. H. Baek, C. B. Eom, and R. Ramesh, Nature Mater. 5, 823 (2006).

${ }^{5}$ R. Ramesh and N. A. Spaldin, Nature Mater. 6, 21 (2007).

${ }^{6}$ Y. H. Chu, L. W. Martin, M. B. Holcomb, M. Gajek, S.-J. Han, Q. He, N. Balke, C.-H. Yang, D. Lee, W. Hu, Q. Zhan, W. Hu, P. L. Yang, A Fraile-Rodriguez, A. Scholl, S. X. Wang, and R. Ramesh, Nature Mater 7, 478 (2008).

${ }^{7}$ C. W. Nan, M. I. Bichurin, S. X. Dong, D. Viehland, and G. Srinivasan, J. Appl. Phys. 103, 031101 (2008).

${ }^{8}$ S. V. Kiselev, R. P. Ozerov, and G. S. Zhdanov, Sov. Phys. Dokl. 7, 742 (1963).

${ }^{9}$ J. R. Teague, R. Gerson, and W. J. James, Solid State Commun. 8, 1073 (1970).

${ }^{10}$ C. Michel, J. M. Moreau, G. D. Achenbach, R. Gerson, and W. J. James, Solid State Commun. 7, 701 (1969).

${ }^{11}$ F. Kubel and H. Schimid, Acta Crystallogr., Sect. B: Struct. Sci. 46, 698 (1990).

${ }^{12}$ P. Fischer, M. Polomska, I. Sosnowska, and M. Szymanski, J. Phys. C 13, 1931 (1980)

${ }^{13}$ C. Ederer and N. A. Spaldin, Phys. Rev. B 71, 060401(R) (2005).

${ }^{14}$ D. Lebeugle, D. Colson, A. Forget, M. Viret, A. M. Bataille, and A. Gukasov, Phys. Rev. Lett. 100, 227602 (2008).

${ }^{15}$ S. Lee, W. Ratcliff, S. W. Cheong, and V. Kiryukhin, Appl. Phys. Lett. 92, 192906 (2008).

${ }^{16}$ M. P. Cruz, Y. H. Chu, J. X. Zhang, P. L. Yang, F. Zavaliche, Q. He, P. Shafter, L. Q. Chen, and R. Ramesh, Phys. Rev. Lett. 99, 217601 (2007).

${ }^{17}$ J. X. Zhang, Y. L. Li, S. Choudhury, L. Q. Chen, Y. H. Chu, F. Zavaliche, M. P. Cruz, R. Ramesh, and Q. X. Jia, J. Appl. Phys. 103, 094111 (2008).

${ }^{18}$ Y. C. Shu and J. H. Yen, Appl. Phys. Lett. 91, 021908 (2007).

${ }^{19}$ Y. C. Shu and J. H. Yen, Acta Mater. 56, 3969 (2008).

${ }^{20}$ Y. C. Shu, J. H. Yen, H. Z. Chen, J. Y. Li, and L. J. Li, Appl. Phys. Lett. 92, 052909 (2008).

${ }^{21}$ L. J. Li, J. Y. Li, Y. C. Shu, H. Z. Chen, and J. H. Yen, Appl. Phys. Lett. 92, 172504 (2008).

${ }^{22}$ K. Bhattacharya, Continuum Mech. Thermodyn. 5, 205 (1993).

${ }^{23}$ A. DeSimone and R. D. James, J. Mech. Phys. Solids 50, 283 (2002).

${ }^{24}$ J. Y. Li and D. Liu, J. Mech. Phys. Solids 52, 1719 (2004).

${ }^{25}$ Y. C. Shu and K. Bhattacharya, Philos. Mag. B 81, 2021 (2001).

${ }^{26}$ W. F. Brown, Micromagnetics (Wesley, New York, 1963).

${ }^{27}$ Y. C. Shu, M. P. Lin, and K. C. Wu, Mech. Mater. 36, 975 (2004)

${ }^{28}$ L. Q. Chen, Annu. Rev. Mater. Res. 32, 113 (2002).

${ }^{29}$ J. X. Zhang and L. Q. Chen, Philos. Mag. Lett. 85, 533 (2005). 